Reynolds Evan (Orcid ID: 0000-0002-0138-8436)

Utility of bilateral lower extremity NCS in DSP 1

Bilateral nerve conduction studies in the evaluation of distal symmetric polyneuropathy

Janae E Dupuis, MD; Jay Li; Brian C Callaghan, MD, MS; Evan L Reynolds; Zachary N

London, MD

Department of Neurology, University of Michigan, Ann Arbor, Michigan

Address correspondence to Janae Dupuis, MD, 1500 E. Medical Center Drive

1324 Taubman Center SPC 5322, Ann Arbor, MI 48109-5322. Telephone: 734-936-9020. Fax:

734-615-4991. E-mail: janaed@med.umich.edu

Running title: Utility of bilateral lower extremity NCS in DSP

Abstract word count: 148

Article word count: 1,202

We confirm that we have read the Journal's position on issues involved in ethical publication and affirm that this report is consistent with those guidelines.

None of the authors has any conflict of interest to disclose.

Key Words: neuropathy, nerve conduction studies, bilateral, electromyography, symmetry

This is the author manuscript accepted for publication and has undergone full peer review but has not been through the copyediting, typesetting, pagination and proofreading process, which may lead to differences between this version and the Version of Record. Please cite this article as doi: $10.1002 /$ mus.26616

This article is protected by copyright. All rights reserved. 


\section{Bilateral nerve conduction studies in the evaluation of distal symmetric polyneuropathy}

Introduction: Nerve conduction studies are used to aid in the diagnosis of distal symmetric polyneuropathy (DSP). Whether bilateral lower extremity nerve conduction studies (NCS) are needed when evaluating for suspected DSP is unclear.

Methods: We retrospectively analyzed nerve conduction studies from patients who presented to the University of Michigan EMG laboratory between July 1, 2016 and December 31, 2017 with symptoms of DSP to assess agreement and correlation between left and right lower extremity NCS parameters.

Results: We found significant agreement between abnormalities in individual nerve parameters of the left and right lower extremities of 105 patients, most notably in the sural nerve. In the 53 patients with bilateral sural, peroneal, and tibial studies, there was also significant agreement between whether the left and right met electrodiagnostic criteria for DSP $(\kappa=0.77)$.

Discussion: Bilateral lower extremity NCS may have limited utility in the evaluation of suspected DSP.

This article is protected by copyright. All rights reserved. 


\section{Introduction}

Nerve conduction studies are commonly utilized to aid in the diagnosis of distal symmetric polyneuropathy (DSP). In combination with clinical history and physical examination, electrodiagnostic testing may help confirm or refute the diagnosis of polyneuropathy. It also can guide management by providing information about the category, severity, and prognosis of polyneuropathy., ${ }^{1,2}$

There has been an effort to standardize nerve conduction study protocols for suspected DSP. Current research guidelines recommend evaluating the sural sensory and peroneal motor nerve in one lower extremity and using the results to determine if additional studies should be performed. ${ }^{3}$ If any of the nerves studied have an absent response, a nerve conduction study of the contralateral nerve should be performed. The recommendations state that contralateral lower extremity nerve conduction studies may also be performed at the discretion of the examiner, regardless of the findings on the first side.

Despite this last recommendation, little evidence exists to support the use of bilateral nerve conduction studies in the diagnosis of DSP. One study found symmetry in nerve conduction studies throughout the various stages of disease with repeated electrodiagnostic testing. ${ }^{4}$ Despite this finding, the authors concluded that bilateral nerve conduction studies should be used when screening patients for DSP.

Electrodiagnostic consultants would be able to make more appropriate decisions about testing if they knew whether nerve conduction studies of a second limb were likely to change 
their overall interpretation. The purpose of this study was to assess the symmetry between right and left lower extremity nerve conduction studies in a cohort of patients with symptoms of DSP. 


\section{Methods}

The study and its methods were approved by the Institutional Review Board of the University of Michigan.

The study population was obtained retrospectively from the University of Michigan EMGPRO database for studies performed from July 1, 2016 through December 31, 2017. Cases were first filtered based on the final diagnosis code applied by the electrodiagnostic consultant. With the goal of identifying all patients who may have presented with symptoms of DSP including those who ended up with an alternative diagnosis, we included codes for neuropathy, lower extremity plexopathy, lower extremity radiculopathy, lower extremity mononeuropathy, normal study, or indeterminate study.

We reviewed the free text referral reasons and the written histories in the electrodiagnostic reports of cases with these diagnosis codes. Only patients referred for neuropathy, polyneuropathy, or lower extremity sensory changes were included. Among these, patients were included only if their histories included lower extremity numbness, tingling or pain, and there was no mention of unilateral or asymmetric symptoms. Finally, we only included cases in which bilateral sural sensory studies were performed.

Nerve conduction study parameters on sural sensory, peroneal motor, and tibial motor nerves were gathered from patients that satisfied all inclusion criteria. Individual parameters were then classified as normal or abnormal based on published normative values. ${ }^{5,6}$ 
Correlation coefficients and Cohen's kappa were used to determine correlation and degree of agreement between individual nerve conduction parameters of right and left sural, peroneal, and tibial nerves. Similarly, we used Cohen's kappa to assess degree of agreement for each nerve using abnormality in one or more parameters in each nerve to categorize abnormal. Current consensus criteria for the diagnosis of distal symmetric polyneuropathy are an abnormality ( $\geq 99$ th or $\leq 1$ st percentile) of any attribute of nerve conduction in two separate nerves, one of which must be the sural nerve. ${ }^{3}$ The subset of cases that included bilateral sural, peroneal, and tibial responses were analyzed to determine whether each side met these criteria for DSP. For each encounter, each side was categorized as normal or abnormal and the two sides were then compared for degree of agreement. 


\section{Results}

Nerve conduction study parameters were gathered from 105 patients. Of these encounters, 53 studies included bilateral sural sensory, peroneal motor, and tibial motor studies. The demographics of patients were 49\% female; $63.4 \pm 12.0$ (mean \pm standard deviation) years (age); $68.4 \pm 4.3$ inches (height); and $200.7 \pm 50.2$ lbs (weight). Of the encounters, 76 were coded as neuropathy, 19 as normal or indeterminate, 4 as mononeuropathy, and 6 as radiculopathy.

A high degree of agreement was observed for abnormalities of any parameter between the right and left lower extremity for the sural, peroneal, and tibial nerves (Table 1). There was also significant agreement between individual nerve parameters of the right and left lower extremities which was most notable in the sural and tibial nerves. Additional analyses showed significant correlation in individual nerve parameters between sides across all three nerves. When we analyzed the subset of electrodiagnostic encounters with bilateral studies of all three nerves to assess criteria for DSP, we found agreement between sides in 89\% (47/53) of cases. ${ }^{3}$ Of the 47 patients with symmetry, 23 had bilateral polyneuropathy and 24 did not meet criteria for polyneuropathy on either side. 


\section{Discussion}

In patients with symmetric signs and symptoms of neuropathy, there was a high degree of agreement in nerve conduction studies between lower extremities including abnormalities between individual nerves and in meeting criteria for DSP. In the correct clinical context, nerve conduction studies on a second lower extremity may not add diagnostic value. Electrodiagnostic testing is one of the most frequently used tests when evaluating for suspected DSP. ${ }^{2}$ Conversely, DSP is among the most common reasons for referral for electrodiagnostic testing. ${ }^{1,7}$ This has practical implications for electrodiagnostic consultants and for neuropathy research. Limiting lower extremity testing to one limb in this population may reduce cost, minimize patient discomfort, and improve provider efficiency.

There are several limitations of our study. Of the 105 patients included, only 53 had bilateral sural, peroneal, and tibial studies which limited our ability to assess symmetry of polyneuropathy between sides. The normative values used in the study were not adjusted for age. We also did not assess symmetry of F-waves which are an important parameter when testing for polyneuropathy.

It should also be noted that our study was done at a large tertiary referral center. If we expanded our study beyond our center, we may have seen a difference in agreement due to variation in clinical practice and electrodiagnostic testing. Another limitation of the study is that the clinical decision making that lead to these patients having bilateral lower extremity nerve conduction studies is unknown. One possibility is that some providers at our institution routinely 
evaluate suspected neuropathy with bilateral nerve conduction studies, while others do not, but we did not gather provider data to evaluate this theory. It is also conceivable that there were undocumented clinical features that led providers to study both lower extremities in certain cases and not others. Future work could survey electrodiagnostic consultants to better determine practice patterns in the community.

Though we demonstrated significant agreement between sides, it is difficult to determine what degree of agreement is enough to justify performing only unilateral nerve conduction studies. Future research could assess how the finding of asymmetry in nerve conduction studies affects clinical decisions in this population.

This single-center study demonstrates that patients with symmetric neuropathy symptoms have a high degree of agreement in nerve conduction study parameters between sides. Given the limitations of a retrospective, single-center study, prospective studies in multiple centers are needed to inform future guidelines about the utility of bilateral nerve conduction studies. 


\section{Abbreviations}

DSP

distal symmetric polyneuropathy

NCS

nerve conduction study

This article is protected by copyright. All rights reserved. 


\section{References}

1. AANEM policy statement on electrodiagnosis for distal symmetric polyneuropathy. Muscle \& Nerve 2017;57(2):337-339. DOI: 10.1002/mus.26003.

2. Bodofsky EB, Carter GT, England JD. Is electrodiagnostic testing for polyneuropathy overutilized? Muscle \& Nerve 2016;55(3):301-304. DOI: 10.1002/mus.25464.

3. England JD, Gronseth GS, Franklin G, Miller RG, Asbury AK, Carter GT, et al. Distal symmetrical polyneuropathy: Definition for clinical research. Muscle \& Nerve 2005;31(1):113123. DOI:10.1002/mus.20233.

4. Perkins BA, Ngo M, Bril V. Symmetry of nerve conduction studies in different stages of diabetic polyneuropathy. Muscle \& Nerve 2002;25(2):212-217. DOI:10.1002/mus.10044.

5. Chen, S, Andary, M, Buschbacher R, Del Toro D, Smith B, So Y, et al. Electrodiagnostic reference values for upper and lower limb nerve conduction studies in adult populations. Muscle \& Nerve 2016;54(3):371-377. DOI:10.1002/mus.25203.

6. Dillingham T, Chen S, Andary M, Buschbacher R, Del Toro D, Smith B, et al. Establishing high-quality reference values for nerve conduction studies: a report from the normative data task force of the American Association of Neuromuscular \& Electrodiagnostic Medicine. Muscle \& Nerve 2016;54:366-370. DOI: 10.1002/mus.25204.

7. Karadag YS, Golgeleyen D, Saka M., Bilen S, Oztekin N, Ak F. Referral Diagnosis Versus Electroneurophysiological Findings-Three Years Experience from a Tertiary Hospital. European Journal of General Medicine 2014;11(4):244-247. DOI:10.15197/sabad.1.11.80.

This article is protected by copyright. All rights reserved. 
Utility of bilateral lower extremity NCS in DSP 12

This article is protected by copyright. All rights reserved. 
Table 1. Correlation and agreement between right and left lower extremity

\begin{tabular}{|c|c|c|c|c|c|c|}
\hline Nerve & $\mathbf{N}$ & Kappa & p-value & $\mathbf{N}$ & Correlation & p-value \\
\hline Sural & 105 & 0.76 & $<0.01$ & & & \\
\hline Amplitude & 105 & 0.79 & $<0.01$ & 105 & 0.94 & $<0.01$ \\
\hline Latency & 105 & 0.85 & $<0.01$ & 105 & 0.79 & $<0.01$ \\
\hline Conduction velocity & 105 & 0.77 & $<0.01$ & 105 & 0.78 & $<0.01$ \\
\hline Peroneal & 65 & 0.75 & $<0.01$ & & & \\
\hline Amplitude & 70 & 0.68 & $<0.01$ & 70 & 0.75 & $<0.01$ \\
\hline Latency & 69 & 0.54 & $<0.01$ & 54 & 0.69 & $<0.01$ \\
\hline Conduction velocity & 65 & 0.77 & $<0.01$ & 51 & 0.86 & $<0.01$ \\
\hline Tibial & 58 & 0.76 & $<0.01$ & & & \\
\hline Amplitude & 60 & 0.74 & $<0.01$ & 60 & 0.85 & $<0.01$ \\
\hline Latency & 59 & 0.81 & $<0.01$ & 54 & 0.69 & $<0.01$ \\
\hline Criteria for $\mathbf{D S P}^{3}$ & 53 & 0.77 & $<0.01$ & & & \\
\hline
\end{tabular}

This article is protected by copyright. All rights reserved. 